\title{
The Scattering Map in the Planar Restricted Three Body Problem
}

\author{
E. Canalias ${ }^{1} \quad$ A. Delshams ${ }^{2} \quad$ J.J. Masdemont ${ }^{3}$ \\ P. Roldán ${ }^{4}$ \\ Departament de Matemtica Aplicada I, ETSEIB-UPC. \\ Avda. Diagonal 647, 08028 Barcelona, Spain. \\ 1 Elisabet.Canalias@upc.edu \\ 2 Amadeu.Delshams@upc.edu \\ 3 josep@barquins.upc.edu \\ ${ }^{4}$ Pablo.Roldan@upc.edu
}

November 15, 2005

\begin{abstract}
We study homoclinic transport to Lyapunov orbits around a collinear libration point in the planar restricted three body problem. A method to compute homoclinic orbits is first described. Then we introduce the scattering map for this problem (defined on a suitable normally hyperbolic invariant manifold) and we show how to compute it using the information already obtained for the homoclinic orbits. An example application to Astrodynamics is also proposed.
\end{abstract}

Keywords: Restricted Three body problem, homoclinic orbits, Normally Hyperbolic Invariant Manifolds, Scattering Map.

\section{Introduction}

Interest in the dynamics around the Lagrangian points has been increasing in the last decades, as $\mathrm{L}_{1}$ and $\mathrm{L}_{2}$ of the Sun-Earth and Earth-Moon systems have been selected for a wide variety of spatial missions (see [1], [6], [7], [3]). The introduction of invariant manifolds as a means to describe the phase space around these points yields not only a more efficient determination of the desired transfers but also an adaptable procedure for mission analysis. Invariant manifolds can be seen as tubes which dominate the dynamics and mass transport in the studied system and in the whole Solar System. In this context, homoclinic trajectories provide zero cost transfers from a libration orbit around $\mathrm{L}_{i}(i=1,2)$ to another one. In the planar case, there is only one periodic motion around 
$\mathrm{L}_{1}$. In particular, homoclinic trajectories are not a means to go from one orbit to another one, but to leave the periodic orbit at a certain point and approach it again in a different point. This feature has possible astrodynamical applications, as the placement of different satellites in previously chosen points of the same orbit.

This paper includes a detailed explanation of the numerical method used for finding homoclinic connections by intersecting the stable and unstable manifold of planar Lyapunov orbits around $\mathrm{L}_{1}$, which was presented for the first time in [2].

Further, we look at the set of Lyapunov orbits around a collinear libration point as a normally hyperbolic invariant manifold $\Lambda$, with associated stable and unstable invariant manifolds $W_{\Lambda}^{s}, W_{\Lambda}^{u}$. These manifolds are important because they organize the dynamics of the system. The hyperbolic invariant manifolds $W_{\Lambda}^{s}, W_{\Lambda}^{u}$ intersect transversally along families of homoclinic orbits $H^{j, i}$.

Following [5], we define the scattering map $S^{j, i}: \Lambda \mapsto \Lambda$ associated to a family of homoclinic orbits. The scattering map relates two trajectories in $\Lambda$ through a homoclinic orbit in the family $H^{j, i}$.

Exploiting the fact that there is only one Lyapunov orbit around the libration point for each value of the Jacobi constant, we show that the scattering map for the planar restricted three body problem has a simple form. Specifically, it is an integrable twist map.

Using only three points from each homoclinic orbit in the family $H^{j, i}$, we are able to compute the associated scattering map. For illustration purposes, in this paper we compute the scattering map for several families of homoclinic orbits to the $L_{1}$ libration point in the Earth-Moon model.

Our future goal is to give a complete description of homoclinic and heteroclinic phenomena in the spatial RTBP. This paper is a first step towards that goal, where we have used the planar restricted three body problem as a test case for the concepts and methods involved.

\section{Methodology}

The RTBP (restricted three body problem) is a model to study the motion of an infinitesimal particle, affected by the gravitational attraction of two massive bodies (primaries), which describe circular orbits around their common center of masses. When the movement of the massless particle is only studied in the plane of motion of the primaries, the problem becomes planar (PRTBP).

Taking adequate units of mass, length and time we can simplify the equations of motion. Furthermore, the following synodical system of reference is taken: the origin set at the center of masses of the two primaries, the $\mathrm{X}$ axis given by the line that goes from the smallest to the biggest primary (with this orientation) and the $\mathrm{Y}$ axis taken so that $\mathrm{XY}$ is a planar positively oriented coordinate system. In our synodical system the primary of mass $\mu$ (small) is always located at $(\mu-1,0)$ and the primary of mass $1-\mu$ at $(\mu, 0)([10])$. The equations of motion are: 


$$
\left.\begin{array}{l}
\ddot{x}-2 \dot{y}=\frac{\partial \Omega}{\partial x} \\
\ddot{y}+2 \dot{x}=\frac{\partial \Omega}{\partial y}
\end{array}\right\}
$$

where,

$$
\begin{array}{r}
\Omega(x, y)=\frac{1}{2}\left(x^{2}+y^{2}\right)+\frac{1-\mu}{r_{1}}+\frac{\mu}{r_{2}}+\frac{1}{2} \mu(1-\mu) . \\
r_{1}^{2}=(x-\mu)^{2}+y^{2}, \quad r_{2}^{2}=(x+1-\mu)^{2}+y^{2} .
\end{array}
$$

The PRTBP in synodical coordinates has five equilibrium points. Three of them are located on the $\mathrm{X}$ axis, and are called Eulerian or collinear points $\left(\mathrm{L}_{1,2,3}\right) . \mathrm{L}_{4}$ and $\mathrm{L}_{5}$ (Lagrangian points) are found as the third vertex of the two equilateral triangles that can be formed using the primaries as vertices (figure 1).

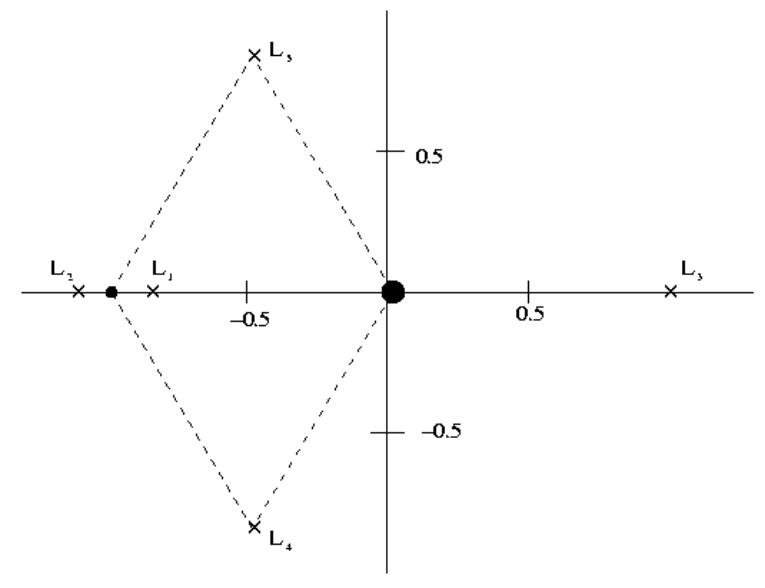

Figure 1: The five equilibrium points of the RTBP.

Introducing momenta $\mathrm{p}_{x}=\dot{x}-y$ and $\mathrm{p}_{y}=\dot{y}+x$, the PRTBP equations of motion cast into a Hamiltonian system. The Hamiltonian function is,

$$
\mathcal{H}\left(x, y, p_{x}, p_{y}\right)=\frac{1}{2}\left(p_{x}^{2}+p_{y}^{2}\right)+y p_{x}-x p_{y}-\frac{1-\mu}{r_{1}}-\frac{\mu}{r_{2}}-\frac{1}{2} \mu(1-\mu)
$$

We define the Jacobi constant, $\mathcal{C}$,

$$
\mathcal{C}(x, y, \dot{x}, \dot{y})=-\left(\dot{x}^{2}+\dot{y}^{2}\right)+2 \Omega(x, y)
$$

with $\Omega$ as in (1). It is easily proved that $\mathcal{C}=-2 \mathcal{H}$. Consequently, the Jacobi constant is a first integral of the RTBP.

In this work we will focus on the dynamics around the equilibrium point $\mathrm{L}_{1}$. The linear behavior of this point is of the type center $\times$ saddle. The 
hyperbolicity introduced by the saddle component is inherited by all the orbits in a big vicinity of $L_{1}$. Moreover, it is responsible for the existence of unstable and stable manifolds arising from the aforementioned orbits.

\subsection{Planar Lyapunov orbits}

For each value of the Jacobi constant, there is a unique planar Lyapunov orbit homeomorphic to $S^{1}$ around $\mathrm{L}_{1}$. This is the unique periodic motion around the $\mathrm{L}_{1}$ for the planar case, and the one we use in this work.

Planar Lyapunov orbits and their hyperbolic manifolds can be computed using Lindstedt-Poincaré procedures. In this way one obtains their expansions in convenient RTBP coordinates.

To this end, we set the origin of coordinates at the libration point and scale the variables in such a way that the distance from the equilibrium point to the small primary is equal to one. The expansion of the equations of motion (1) in these variables $(\bar{x}, \bar{y})$ takes the form,

$$
\left\{\begin{aligned}
\ddot{\bar{x}}-2 \dot{\bar{y}}-\left(1+2 c_{2}\right) \bar{x} & =\frac{\partial}{\partial \bar{x}} \sum_{n \geq 3} c_{n} \rho^{n} P_{n}\left(\frac{\bar{x}}{\rho}\right) \\
\ddot{\bar{y}}+2 \dot{\bar{x}}+\left(c_{2}-1\right) \bar{y} & =\frac{\partial}{\partial \bar{y}} \sum_{n \geq 3} c_{n} \rho^{n} P_{n}\left(\frac{\bar{y}}{\rho}\right)
\end{aligned}\right.
$$

where $\rho^{2}=\bar{x}^{2}+\bar{y}^{2}, P_{n}$ is the Legendre polynomial of degree $n$, and $c_{n}$ are constants which depend only on $\mu$ and the selected equilibrium point (see [8]). We note that in (3) the linear terms appear in the left hand side part of the equations and the nonlinear ones in the right hand side. The solution of the linear part of the equations (3) is:

$$
\begin{aligned}
& \bar{x}_{1}(t)=\alpha_{1} \exp \left(\lambda_{0} t\right)+\alpha_{2} \exp \left(-\lambda_{0} t\right)+\alpha_{3} \cos \left(\omega_{p} t+\phi\right), \\
& \bar{y}_{1}(t)=\kappa_{2} \alpha_{1} \exp \left(\lambda_{0} t\right)-\kappa_{2} \alpha_{2} \exp \left(-\lambda_{0} t\right)+\kappa_{1} \alpha_{3} \sin \left(\omega_{p} t+\phi\right)
\end{aligned}
$$

where $\kappa_{1}, \kappa_{2}, \omega_{p}$ and $\lambda_{0}$ are constants for a given model.

The $\alpha$ 's are free amplitudes. $\alpha_{1}$ and $\alpha_{2}$ are the ones associated with the hyperbolic manifolds. If $\alpha_{1}=\alpha_{2}=0$, we have the linear part of the Lyapunov orbit with amplitude $\alpha_{3}$. When $\alpha_{1}=0$ and $\alpha_{2} \neq 0$ we have orbits tending to the Lyapunov orbit of amplitude $\alpha_{3}$ when time tends to infinity (stable manifold). On the contrary when $\alpha_{2}=0$ and $\alpha_{1} \neq 0$, orbits leave the vicinity of the Lyapunov exponentially fast in forward time (unstable manifold).

When we consider also the non-linear terms of (3), solutions close to $\mathrm{L}_{1}$ are obtained by means of formal series in powers of the amplitudes of the form:

$$
\begin{aligned}
& \bar{x}(t)=\sum e^{(i-j) \theta_{2}}\left[x_{i j k}^{p} \cos \left(p \theta_{1}\right)+\bar{x}_{i j k}^{p} \sin \left(p \theta_{1}\right)\right] \alpha_{1}^{i} \alpha_{2}^{j} \alpha_{3}^{k} \\
& \bar{y}(t)=\sum e^{(i-j) \theta_{2}}\left[y_{i j k}^{p} \cos \left(p \theta_{1}\right)+\bar{y}_{i j k}^{p} \sin \left(p \theta_{1}\right)\right] \alpha_{1}^{i} \alpha_{2}^{j} \alpha_{3}^{k}
\end{aligned}
$$

where $\theta_{1}=\omega t+\phi, \theta_{2}=\lambda t$, and $\omega=\sum \omega_{i j k} \alpha_{1}^{i} \alpha_{2}^{j} \alpha_{3}^{k}, \lambda=\sum \lambda_{i j k} \alpha_{1}^{i} \alpha_{2}^{j} \alpha_{3}^{k}$. Summation is extended over all $i, j, k$ and $p \in \mathbb{N}$. 
However, due to symmetries, many of the coefficients $x_{i j k}^{p}, \bar{x}_{i j k}^{p}, y_{i j k}^{p}, \bar{y}_{i j k}^{p}, \omega_{i j k}, \lambda_{i j k}$ are zero. It is important to note that for the frequency series, the coefficient $\omega_{i j k} \neq 0$ only if $i=j$. Thus, we can write,

$$
\omega=\sum \omega_{l k}\left(\alpha_{1} \alpha_{2}\right)^{l} \alpha_{3}^{k}, \quad \lambda=\sum \lambda_{l k}\left(\alpha_{1} \alpha_{2}\right)^{l} \alpha_{3}^{k}
$$

In the computations, the series are truncated at a certain (high) order ([8]). Nevertheless, we note that the meaning of the amplitudes in the nonlinear expansions (5) is the same one as in the linear solutions (4).

Therefore, in these coordinates, we can write the Lyapunov orbit of amplitude $\alpha$ as,

$$
\lambda_{\alpha}=\left\{x=\left(\alpha_{1}, \alpha_{2}, \alpha_{3}, \phi\right) \in \mathbb{R}^{3} \times \mathbb{T}: \alpha_{1}=\alpha_{2}=0, \alpha_{3}=\alpha, \phi \in \mathbb{T}\right\} .
$$

Notice that in the planar restricted three body problem there is a bijective correspondence between values of the Jacobi constant and values of the amplitude $\alpha_{3}$. Thus we can also consider coordinates $x=\left(\alpha_{1}, \alpha_{2}, \mathcal{C}, \phi\right) \in \mathbb{R}^{3} \times \mathbb{T}$ and write the above Lyapunov orbit as

$$
\lambda_{C}=\left\{x=\left(\alpha_{1}, \alpha_{2}, \alpha_{3}, \phi\right) \in \mathbb{R}^{3} \times \mathbb{T}: \alpha_{1}=\alpha_{2}=0, \mathcal{C}=C, \phi \in \mathbb{T}\right\}
$$

where $C$ is the Jacobi constant associated to $\alpha$.

We can now define the local unstable and stable manifolds associated to $\lambda_{C}$ :

$$
\begin{aligned}
& W_{\lambda_{C}, \text { loc }}^{s}=\left\{\left(0, \alpha_{2}, C, \phi\right): \alpha_{2} \in \mathbb{R}, \phi \in \mathbb{T}\right\} \\
& W_{\lambda_{C}, \text { loc }}^{u}=\left\{\left(\alpha_{1}, 0, C, \phi\right): \alpha_{1} \in \mathbb{R}, \phi \in \mathbb{T}\right\}
\end{aligned}
$$

\subsection{Hyperbolic manifolds}

In the previous section we saw how the Lyapunov orbits and their stable and unstable manifolds can be described semi-analytically up to a certain order. Nevertheless, as far as manifolds are concerned, the analytical description is used only in a small vicinity of the Lyapunov orbit. For the rest of the tube, the manifold is obtained by numerically integrating this semi-analytical expression.

That is, there are two different parts in the description of each manifold. By setting $\alpha_{1}= \pm \epsilon$ and $\alpha_{2}=0$ (respectively $\alpha_{2}= \pm \epsilon$ and $\alpha_{1}=0$ ), and the rest of the amplitudes and phases fixed to the chosen Lyapunov orbit values, a point on the local unstable (respectively, stable) invariant manifold is obtained in the series (5). Set $t=0$, so we can refer to these points as initial conditions. Furthermore, the aforementioned initial conditions are at a distance of order $\epsilon$ from the Lyapunov orbit. The part of the local manifold which asymptotically joins them with the Lyapunov orbit is described analytically by the same series for values of time $t \neq 0$. In the case $\alpha_{1} \neq 0$, negative values of time tending to $-\infty$ result in points belonging to the local unstable manifold and approaching 


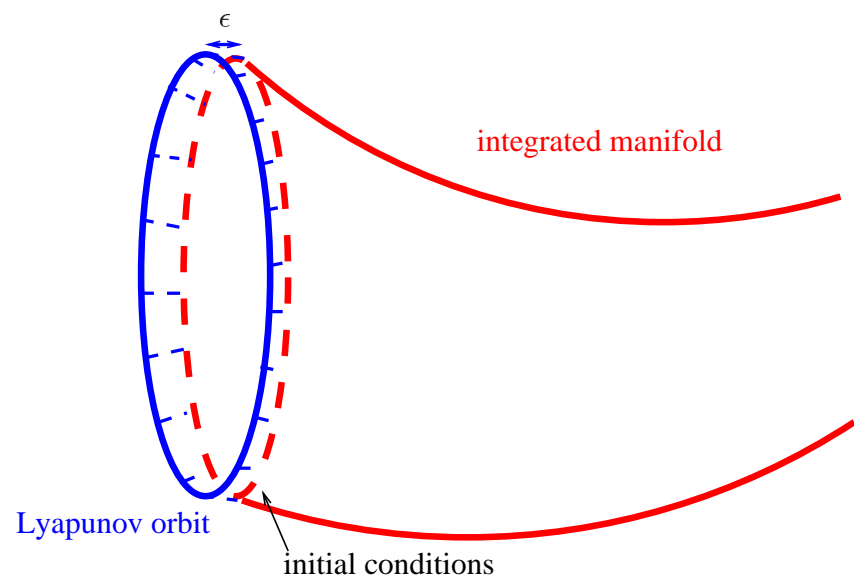

Figure 2: Two different parts in the description of the Hyperbolic Manifolds: analytical series and numerical integration.

the Lyapunov asymptotically (respectively, positive times tending to $+\infty$ for $\left.\alpha_{1} \neq 0\right)$. This part is represented, schematically, in figure 2 as the small gap between the 'Lyapunov orbit' and the 'initial conditions'.

For other values of time (positive for the unstable case and negative for the stable), the manifold tubes can be continued by integrating the initial conditions: in forward time for the case $\alpha_{1} \neq 0$ and backwards in time for $\alpha_{2} \neq 0$ (part labelled as 'integrated manifold' in figure 2). Note that this numerical integration is necessary, as it provides us with an accurate description of the manifolds in regions where the Lindstedt-Poincaré expansions are no longer valid due to the big distance to the equilibrium point.

\subsection{Numerical computation of homoclinic trajectories}

In order to look for homoclinic orbits of a Lyapunov orbit emerging from $\mathrm{L}_{1}$, we will use a Poincare section. This is a common technique in the study of dynamical systems, and consists of observing the flow of the system only in the points that cross a given section. In this way, the flow is studied in a lower dimensional space, but relevant information can still be derived. The stable and unstable manifolds of Lyapunov planar orbits are 2-dimensional objects (see section 2.2). If we study them only in their cut with a Poincaré section (i.e. $\mathcal{S}=\{(x, y, \dot{x}, \dot{y}): x=K\}$, where $K$ is a constant), the resulting objects are closed curves, and therefore, much easier to study and represent. These two closed curves may intersect transversally in several points. Each intersection point belongs to a different homoclinic connection to the Lyapunov orbit.

One can consider the first, second, and so on, cut of the invariant manifolds with the Poincaré section, and obtain in this way different closed curves which 
may have a different number of intersections and therefore, a different number of homoclinic connections (which perform several loops around the primary).

So, take $\mathcal{S}$ as the plane in the state space of the points with $x=-1+\mu$. Now, the process of finding homoclinic connections, $\gamma_{\mathcal{C}}$, to a Lyapunov orbit, $\lambda_{\mathcal{C}}$, consists of three parts:

1 Integration of the manifolds $\left(W_{\lambda_{\mathcal{C}}}^{s}, W_{\lambda_{\mathcal{C}}}^{u}\right)$ arising from the Lyapunov orbits $\left(\lambda_{\mathcal{C}}\right)$ until they cut the Poincaré section $\mathcal{S}$ (see figure 3 ).

2 Detection of intersections between the cuts of the asymptotic manifolds $\left(W_{\lambda_{\mathcal{C}}}^{s} \cap \mathcal{S}\right.$ and $W_{\lambda_{\mathcal{C}}}^{u} \cap \mathcal{S}$ ) in the Poincaré section (see figure 3 ).

3 Refinement of the intersections up to a certain precision.
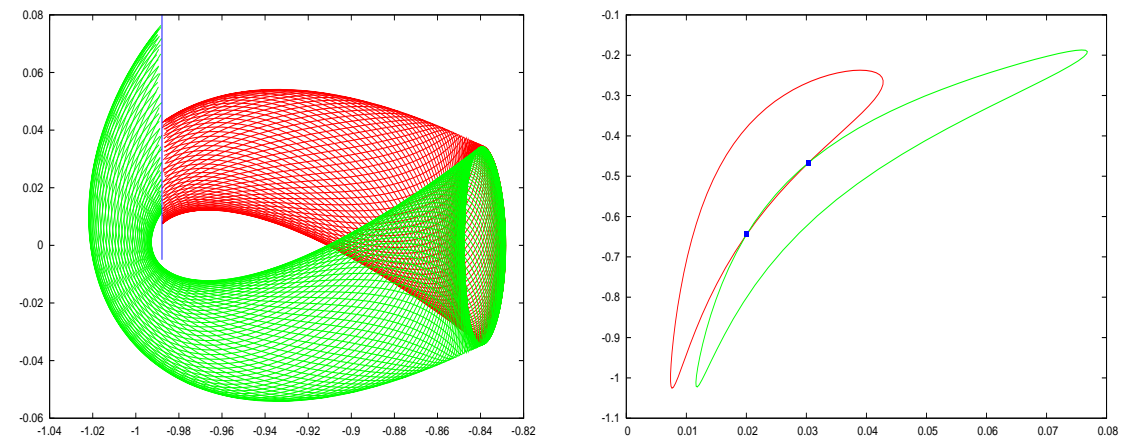

Figure 3: (left) Hyperbolic manifolds of a planar Lyapunov orbit around $L_{1}$. The stable one is integrated until the second cut with the Poincaré section, while the unstable one until the first cut. (right) $y \dot{y}$ representation of the cut of the manifolds on the Poincare section. The blue dots represent the intersecting points between both manifolds, that is the homoclinic trajectories.

\section{Integration of the manifolds}

As it has been said, when the value of $\mathcal{C}$ is chosen, $\alpha_{3}$ is fixed. In order to take initial conditions on the unstable (respectively stable) local manifolds in (5) we set $\alpha_{1}= \pm \epsilon$ (respectively $\left.\alpha_{2}= \pm \epsilon\right) . \epsilon$ is a small parameter $\left(\approx 10^{-3}\right)$, and the sign indicates towards which branch of the manifold we will integrate: In $\mathrm{L}_{1},+\epsilon$ means towards the small primary, while $-\epsilon$ means towards the interior region.

The discretization on the initial conditions arises from taking $n$ phases around the Lyapunov orbit $\lambda_{\mathcal{C}}, \theta_{1}{ }^{i}(0)=\phi^{i}=2 \pi i / n, \quad i=0, \ldots, n-1$.

The bunch of orbits launched by these kind of initial conditions, with the series in (5) truncated at order 15, form manifold tubes. A Runge-Kutta-Fehlberg method of orders 7-8 is then used for the integration. Note that order 15 provides a precise approximation to the manifolds which is adequate in order to 
fulfil rigorous numerical requirements (in particular, enough for the purposes of this work).

As the equation of the Poincaré section is $x=\mu-1$, the number of cuts $(\mathrm{N})$ with the section can be easily controlled by studying the sign of $x(t)+1-$ $\mu$. When $\mathrm{N}$ matches the desired number of cuts, a one dimensional Newton procedure is used in order to obtain the point on the section with an accuracy of at least $10^{-12}$ (i.e. $|x(t)+1-\mu|<10^{-12}$ ).

2. Detection of intersections between the cuts of asymptotic manifolds in the Poincaré section.

Once the integration is completed, we have a set of points on the Poincaré section belonging to a discrete bunch of trajectories lying on the manifolds ( $n$ trajectories if $n$ phases where used). So, the result of joining these points is a polygon made of $n$ segments. The integration is performed for both the unstable manifold and the stable one, so two curves are obtained on the Poincaré section (see the top pictures in figure 3 ).

The next step that has to be taken is to check for intersections on the section $\mathcal{S}$ between each segment of one of the curves and all the segments of the other curve. All in all, $\left(n_{1}\right) \times\left(n_{2}\right) \approx O\left(n^{2}\right)\left(n_{i}\right.$ number of $\theta_{1}$ in manifold $\left.i\right)$ comparisons between segments. This is why it is not worth taking big $n$ values, because it does not enhance the precision of the final cut, but increases the computational effort. Nevertheless, $n$ has to be big enough for the cuts on the Poincaré section to be well determined with an approximated $S^{1}$ structure when the segments are joined together (for instance, $n_{1}=n_{2}=100$ ).

There is a correspondence between each segment $(a, b)$ on the section and a segment $\left(\phi^{a}, \phi^{b}\right) \in \mathbb{T}$, such that $a$ (resp. $b$ ) is the integrated point corresponding to the initial condition on the manifold with $\theta_{1}=\phi^{a}$ (resp. $\phi^{b}$ ) Therefore, at the end of this process we store 4 phase values for each detected cut. Two of them for the unstable manifold, $\left(\phi_{u}^{a}, \phi_{u}^{b}\right)$ and the other two $\left(\phi_{s}^{a}, \phi_{s}^{b}\right)$ for the stable manifold.

\section{Refinement of the intersections.}

We start with the intervals found in the previous step, and we look for $\phi_{u} \in\left[\phi_{u}^{a}, \phi_{u}^{b}\right]$ and $\phi_{s} \in\left[\phi_{s}^{a}, \phi_{s}^{b}\right]$, which define the exact initial conditions of the connecting trajectory.

We use a secant-like method to find the intersection between the segments $s_{u}^{0}=\left(\phi_{u}^{a_{0}}, \phi_{u}^{b_{0}}\right)$ and $s_{s}^{0}=\left(\phi_{s}^{a_{0}}, \phi_{s}^{b_{0}}\right)$, which we know have a non-empty intersection if $a_{0}=a$ and $b_{0}=b$. The pair of intersecting phases divides each of the original segments in two, so 4 new segments are defined. Now we look for intersections between the segments associated with $W_{\lambda_{\mathcal{C}}}^{u}$ and the ones associated with $W_{\lambda_{\mathcal{C}}}^{s}$. Only one of the four checks should be positive. So, we store only the four phases

which are in the border of the new intersecting segments $\mathrm{s}_{u}^{1}$ and $\mathrm{s}_{s}^{1}$. Thus we have again $\left[\phi_{u}^{a_{1}}, \phi_{u}^{b_{1}}\right],\left[\phi_{s}^{a_{1}}, \phi_{s}^{b_{1}}\right]$, but the longitude of the intervals has been reduced.

We iterate the process $k$ times until $\left|s_{u}^{k}\right|+\left|s_{s}^{k}\right|=\left|\phi_{u}^{a_{k}}-\phi_{u}^{b_{k}}\right|+\left|\phi_{s}^{a_{k}}-\phi_{s}^{b_{k}}\right|<$ $10^{-12}$. 
When the three steps have been successfully finished for an adequate $\mathcal{C}$, we have $\left(\phi_{u}, \phi_{s}\right)$, the phases on the Lyapunov orbit defining the connection. We have the intersecting point on the Poincaré section $\mathcal{S}$, and the corresponding integration times as well.

So, the connecting trajectory can be reconstructed by taking the following initial conditions:

- For the unstable manifold: $\left(\alpha_{1}, \alpha_{2}, \alpha_{3}, \theta_{1}\right)=\left(10^{-3}, 0, \alpha_{3}(\mathcal{C}), \phi_{u}\right)$.

- For the stable manifold: $\left(\alpha_{1}, \alpha_{2}, \alpha_{3}, \theta_{1}\right)=\left(0,-10^{-3}, \alpha_{3}(\mathcal{C}), \phi_{s}\right)$

The integration of these initial conditions yields the connection. Obviously, the one on the unstable manifold has to be integrated forwards in time, while the other one backwards.

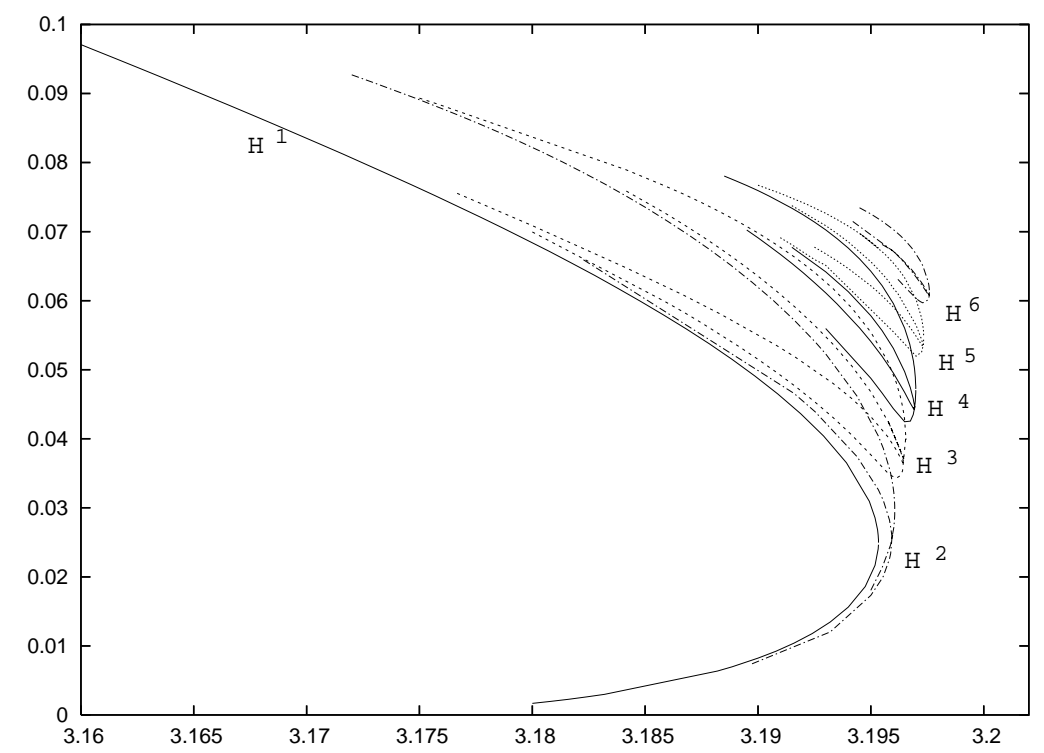

Figure 4: Families of $L_{1}$ homoclinic orbits in the Earth-Moon case.

Once a homoclinic connection to a Lyapunov orbit $\lambda_{\mathcal{C}}$ has been found, some other connecting trajectories can be found to nearby Lyapunov orbits $\lambda_{\mathcal{C}^{\prime}}$ by slightly varying the initial conditions (due to the analytic continuous dependence of the solutions of the dynamical equations w.r.t. these initial conditions). A group of trajectories which are coincident in some of the initial parameters giving rise to one-parameter family of homoclinic trajectories will be called a family of homoclinic trajectories and denoted by $H$. We can classify the families of homoclinic trajectories to $\mathrm{L}_{1}$ planar Lyapunov orbits depending on the number of cuts with the Poincaré section or equivalently, the number of loops, $j$, around the small primary that the trajectories do. In addition, for the same number 
of cuts with the section $\mathcal{S}$, more than one family is obtained. In particular, the number of families is equal to the number of intersections between the manifolds on the section.

To construct these continuous one-parameter families $H^{j, i}$ of homoclinic trajectories, we consider only those intervals $\left(\mathcal{C}_{-}^{j, i}, \mathcal{C}_{+}^{j, i}\right)$ of Jacobi constants for which the intersections between the manifolds are transversal. The specific values $\mathcal{C}_{ \pm}^{j, i}$ are candidates to bifurcation homoclinic intersections.

Thus, we will write,

$$
H^{j, i}=\bigcup_{\mathcal{C} \in\left(\mathcal{C}_{-}^{j, i}, \mathcal{C}_{+}^{j, i}\right)} \gamma_{\mathcal{C}}^{j, i}
$$

where $\left(\mathcal{C}_{-}^{j, i}, \mathcal{C}_{+}^{j, i}\right)$ is the interval of Jacobi constants for which the family is ob-

tained, $j$ is the number of loops and $i$ is the integer identifying the family depending on the $y$ coordinate on the Poincaré section $(i=1$ corresponding to the families yielding the intersection with smallest $y$ ).

Notice that, by construction, for any $j$ and $i$, and any $\mathcal{C} \in\left(\mathcal{C}_{-}^{j, i}, \mathcal{C}_{+}^{j, i}\right)$, there exists a unique homoclinic orbit $\gamma_{\mathcal{C}}^{j, i} \subset H^{j, i}$ to the Lyapunov orbit $\lambda_{\mathcal{C}}$.

Figure 4 is a representation of the families that have been obtained using the procedure explained above for the Earth-Moon mass parameter $\mu$. The notation is simplified in the figure to $H^{j}$. Each one of the branches corresponds to $H^{j, i}$, with $i$ increasing upwards. The $x$-axis in the figures corresponds to the value of the Jacobi constant, and the $y$-axis to the $y$-coordinate of the trajectory in one of the cuts with the Poincaré section in adimensional RTBP coordinates.

\section{$3 \quad$ Scattering Map}

\subsection{The NHIM $\Lambda$ and associated Hyperbolic Manifolds}

In section 2.1, Lindstedt-Poincaré variables for the restricted three body problemaround the fixed point have been introduced. A point in phase space close to $L_{1}$ is written in these variables as $X=\left(\alpha_{1}, \alpha_{2}, \alpha_{3}, \phi\right) \in \mathbb{B} \times \mathbb{T} \subset \mathbb{R}^{3} \times \mathbb{T}$, where $\mathbb{B}=I_{1} \times I_{2} \times I_{3}$ is an open rectangle containing the origin in $\mathbb{R}^{3}$. These variables are convenient because they parametrize the invariant manifolds present in the system in a natural way.

Let $\left[\mathcal{C}_{1}, \mathcal{C}_{2}\right]$ be an interval of energy values such that $\forall \mathcal{C} \in\left[\mathcal{C}_{1}, \mathcal{C}_{2}\right]$ there exists (at least) one orbit homoclinic to $\lambda_{\mathcal{C}}$. Following [5] we introduce the set

$$
\begin{aligned}
\Lambda & =\left\{X \in \mathbb{B} \times \mathbb{T}: \alpha_{1}=\alpha_{2}=0, \mathcal{C} \in\left[\mathcal{C}_{1}, \mathcal{C}_{2}\right]\right\} \\
& =\bigcup_{\mathcal{C} \in\left[\mathcal{C}_{1}, \mathcal{C}_{2}\right]} \lambda_{\mathcal{C}} .
\end{aligned}
$$

This set is a 2-dimensional manifold with boundary. It is invariant for the flow $\Phi_{t}$ of the system. The manifold $\Lambda$ is topologically $\left[\mathcal{C}_{1}, \mathcal{C}_{2}\right] \times \mathbb{T}$. A point in the manifold is parametrized by $(\mathcal{C}, \phi) . \Lambda$ is a subset of the so-called center manifold. 


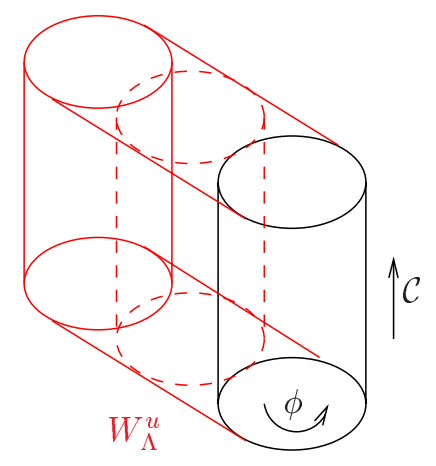

$\Lambda$

Figure 5: Schematic representation of the normally hyperbolic invariant manifold $\Lambda$ and one of its asymptotic manifolds $W_{\Lambda}^{u}$.

Notice that $\Lambda$ is actually a normally hyperbolic invariant manifold for the flow (see [5] for the definition and properties of a normally hyperbolic invariant manifold).

The sets

$$
\begin{aligned}
& W_{\Lambda}^{s}=\bigcup_{\mathcal{C} \in\left[\mathcal{C}_{1}, \mathcal{C}_{2}\right]} W_{\lambda_{\mathcal{C}}}^{s}, \\
& W_{\Lambda}^{u}=\bigcup_{\mathcal{C} \in\left[\mathcal{C}_{1}, \mathcal{C}_{2}\right]} W_{\lambda_{\mathcal{C}}}^{u}
\end{aligned}
$$

are called stable and unstable manifolds of $\Lambda$ in normally hyperbolic theory. They are 3 -dimensional manifolds. A local parametrization is given by

$$
\begin{aligned}
& W_{\Lambda, \text { loc }}^{s}=\left\{\left(0, \alpha_{2}, \mathcal{C}, \phi\right): \alpha_{2} \in I_{2} \subset \mathbb{R}, \mathcal{C} \in\left[\mathcal{C}_{1}, \mathcal{C}_{2}\right], \phi \in \mathbb{T}\right\} \\
& W_{\Lambda, \text { loc }}^{u}=\left\{\left(\alpha_{1}, 0, \mathcal{C}, \phi\right): \alpha_{1} \in I_{1} \subset \mathbb{R}, \mathcal{C} \in\left[\mathcal{C}_{1}, \mathcal{C}_{2}\right], \phi \in \mathbb{T}\right\} .
\end{aligned}
$$

Recall the definition of the stable manifold of a point $X$ in a hyperbolic set $\Lambda$ :

$$
W_{X}^{s}=\left\{Y \in \mathbb{B} \times \mathbb{T}: \operatorname{dist}\left(\Phi_{t}(X), \Phi_{t}(Y)\right) \rightarrow 0, \quad t \rightarrow+\infty\right\} .
$$

In general, this manifold is not invariant for the flow of the system. (It is the nonlinear analog of the contracting subspace $\mathbb{E}_{x}^{s}$ in the hyperbolic splitting of a hyperbolic invariant set. For an introduction to hyperbolic invariant sets, see [9]). However, the stable manifold of a point satisfies the property

$$
\Phi_{t}\left(W_{X}^{s}\right)=W_{\Phi_{t}(X)}^{s} .
$$

In the case of the planar restricted three body problem, the local stable manifold of a point $X_{+}=\left(0,0, \mathcal{C}_{+}, \phi_{+}\right) \in \Lambda$ is given by

$$
W_{X_{+}, \text {loc }}^{s}=\left\{\left(0, \alpha_{2}, \mathcal{C}_{+}, \phi_{+}\right): \alpha_{2} \in I_{2}\right\}
$$


as one can easily check from equations (5) and (6). Analogously, given $X_{-}=$ $\left(0,0, \mathcal{C}_{-}, \phi_{-}\right) \in \Lambda$, its local unstable manifold is given by

$$
W_{X_{-}, \text {loc }}^{u}=\left\{\left(\alpha_{1}, 0, \mathcal{C}_{-}, \phi_{-}\right): \alpha_{1} \in I_{1}\right\} .
$$

It is thus evident that

$$
W_{\Lambda}^{s}=\bigcup_{X \in \Lambda} W_{X}^{s} \quad \text { and } \quad W_{X}^{s} \cap W_{Y}^{s}=\emptyset \quad \text { if } X \neq Y .
$$

There is a similar foliation for the unstable manifold.

\subsection{The dynamics in $\Lambda$}

In the case of the planar restricted three body problem, the dynamics restricted to $\Lambda$ is very simple. The normally hyperbolic invariant manifold is foliated by invariant tori corresponding to periodic Lyapunov orbits. The flow on $\Lambda$ is given by

$$
\Phi_{t}\left(0,0, \mathcal{C}_{0}, \phi_{0}\right)=\left(0,0, \mathcal{C}_{0}, \phi_{0}+\omega t\right)
$$

where $\omega$ is given in (6), and we will denote by

$$
\lambda_{t}\left(\mathcal{C}_{0}, \phi_{0}\right)=\left(0,0, \mathcal{C}_{0}, \phi_{0}+\omega t\right)
$$

the orbits in the torus $\lambda_{\mathcal{C}_{0}}$.

\subsection{Definition of the Scattering Map}

In this section we are going to define a scattering map $S^{j, i}$ for every family $H^{j, i}$ of homoclinic orbits to $\Lambda$. For ease of presentation, through this section we will drop the indices that identify a homoclinic family.

Consider a homoclinic family $H$ defined on a given interval $\left(\mathcal{C}_{-}, \mathcal{C}_{+}\right)$. Take a point $Z^{*} \in H$ and let $\mathcal{C} \in\left(\mathcal{C}_{-}, \mathcal{C}_{+}\right)$be its Jacobi constant, so that $Z^{*} \in \gamma_{\mathcal{C}}$. By definition of the stable and unstable manifolds of $\Lambda$ (see (10)), there exist $X_{-}^{*}, X_{+}^{*} \in \Lambda$ such that

$$
\operatorname{dist}\left(\Phi_{t}\left(Z^{*}\right), \Phi_{t}\left(X_{ \pm}^{*}\right)\right) \rightarrow 0 \quad t \rightarrow \pm \infty,
$$

which are unique by (11). Indeed, by preservation of the Jacobi constant, $X_{-}^{*}, X_{+}^{*} \in \lambda_{\mathcal{C}}$.

Consider now an arbitrary point $X_{-}$in the Lyapunov periodic orbit $\lambda_{\mathcal{C}}$, so that $X_{-}=\Phi_{\tau}\left(X_{-}^{*}\right)$, with $\tau \in\left[0, T_{\mathcal{C}}\right)$, where $T_{\mathcal{C}}$ is the period of $\lambda_{\mathcal{C}}$. We define $X_{+}=\Phi_{\tau}\left(X_{+}^{*}\right) \in \lambda_{\mathcal{C}} \subset \Lambda, Z=\Phi_{\tau}\left(Z^{*}\right) \in \gamma_{\mathcal{C}}$ and notice that

$$
\operatorname{dist}\left(\Phi_{t}(Z), \Phi_{t}\left(X_{ \pm}\right)\right) \rightarrow 0 \quad t \rightarrow \pm \infty .
$$

The map

$$
X_{-} \in \mathcal{D} \subset \Lambda \stackrel{S}{\longmapsto} X_{+} \in \mathcal{D} \subset \Lambda
$$


where $\mathcal{D}=\cup_{\mathcal{C} \in\left(\mathcal{C}_{-}, \mathcal{C}_{+}\right)} \lambda_{\mathcal{C}}$, is called the scattering map and is characterized by the property that

$$
X_{+}=S\left(X_{-}\right) \text {if and only if there exists } Z \in H \text { such that (17) holds. }
$$

Notice that the scattering map $S=S^{j, i}$ and its domain of definition $\mathcal{D}=\mathcal{D}^{j, i}$ depend on the homoclinic family $H^{j, i}$.

Next we check this characterization. Assume that there exist $Z_{1}, Z_{2} \in H$ and $X_{+, 1}, X_{+, 2} \in \Lambda$ with

$$
\operatorname{dist}\left(\Phi_{t}\left(Z_{k}\right), \Phi_{t}\left(X_{-}\right)\right) \rightarrow 0 \quad t \rightarrow-\infty
$$

but

$$
\operatorname{dist}\left(\Phi_{t}\left(Z_{k}\right), \Phi_{t}\left(X_{+, k}\right)\right) \rightarrow 0 \quad t \rightarrow+\infty .
$$

Since $Z_{1}, Z_{2}$ belong to $\gamma_{\mathcal{C}}, Z_{2}=\Phi_{t_{1}}\left(Z_{1}\right)$, and imposing (19) and the fact that $\lambda_{\mathcal{C}}$ is periodic, one easily gets that $t_{1}=n T_{\mathcal{C}}$ for some integer $n$. Imposing now (20), one finds that $X_{+, 1}=X_{+, 2}$. This proves that the scattering map $S$ is well defined and that it does not depend on the choice of the point $Z$ in (17). Analogously, one can check that $S^{-1}: X_{+} \mapsto X_{-}$is also well defined.

An interesting property of the scattering map is that for any $t \in \mathbb{R}$

$$
S\left(\Phi_{t}\left(X_{-}\right)\right)=\Phi_{t}\left(X_{+}\right),
$$

which follows readily from the characterization (17).

We now look for the expression of the scattering map in the coordinates $(\mathcal{C}, \phi)$ of $\Lambda$ introduced in (8).

Due to the low dimensional nature of the planar restricted three body problem, one can easily describe the scattering map associated to a homoclinic manifold. Let $X_{-}, X_{+} \in \Lambda$ have Lindstedt-Poincaré variables

$$
\begin{aligned}
& X_{-}=\left(\mathcal{C}, \phi_{-}\right), \\
& X_{+}=\left(\mathcal{C}, \phi_{+}\right),
\end{aligned}
$$

so that $S\left(\mathcal{C}, \phi_{-}+\omega t\right)=\left(\mathcal{C}, \phi_{+}+\omega t\right)$ by property $(21)$. Introduce the phase shift

$$
\Delta=\Delta(\mathcal{C})=\phi_{+}-\phi_{-}
$$

which depends on the torus. Then we can express the scattering map as

$$
S(\mathcal{C}, \phi)=(\mathcal{C}, \phi+\Delta(\mathcal{C}))
$$

for any $(\mathcal{C}, \phi) \in\left(\mathcal{C}_{-}, \mathcal{C}_{+}\right) \times \mathbb{T}$. Hence the scattering map has the form of an integrable twist map. As a matter of fact, it is a nontrivial twist map, that is $\Delta^{\prime}(\mathcal{C}) \not \equiv 0$, as will be shown in the next section. 


\subsection{Computation of the Scattering Map}

As explained in the previous section, the particular geometrical features of the planar restricted three body problem impose a strong structure for the scattering map associated to a homoclinic manifold. For every $j, i$,

- $S=S^{j, i}$ can be computed independently on each torus $\lambda_{\mathcal{C}} \in \mathcal{D}^{j, i}$.

- On a given torus $\lambda_{\mathcal{C}}$, the scattering map is just a translation $\phi \mapsto \phi+\Delta$ by a constant shift $\Delta=\Delta^{j, i}(\mathcal{C})$.

Therefore, the problem of computing $S$ reduces to computing $\Delta(\mathcal{C})$ for any torus in the range $\mathcal{C} \in\left(\mathcal{C}_{-}^{j, i}, \mathcal{C}_{+}^{j, i}\right)$, where $\Delta(\mathcal{C})$ is given in $(22)$.

Remark 3.1 The characterization of the scattering map in terms of a scalar function $\Delta(\mathcal{C})$ is very specific of this problem, because $\mathcal{C}$ is a constant of motion and there is only one torus $\lambda_{\mathcal{C}}$ for every $\mathcal{C}$. Obviously, this will not happen in the spatial restricted three body problem, where the $\lambda_{\mathcal{C}}$ have to be replaced by 2-dimensional KAM invariant tori.

Let us fix $\mathcal{C} \in\left(\mathcal{C}_{-}^{j, i}, \mathcal{C}_{+}^{j, i}\right)$ and consider the corresponding 1-torus $\lambda_{\mathcal{C}}$ and homoclinic orbit $\gamma_{\mathcal{C}}=\gamma_{\mathcal{C}}^{j, i}$. We seek $X_{-}=\left(\mathcal{C}, \phi_{-}\right)$and $X_{+}=\left(\mathcal{C}, \phi_{+}\right)$in $\lambda_{\mathcal{C}}$ such that

$$
Z \in W_{X_{-}}^{u}
$$

and

$$
Z \in W_{X_{+}}^{s}
$$

for some $Z \in \gamma_{\mathcal{C}}$.

Note that $X_{-}$and $X_{+}$are related to $Z$ asymptotically, so these two points cannot be found from $Z$ by direct iteration. The algorithm used in section 2.3 to find the homoclinic connection $\gamma_{\mathcal{C}}$ provides us with $X_{u} \in W_{\lambda_{\mathcal{C}}}^{u}, X_{s} \in W_{\lambda_{\mathcal{C}}}^{s}$ and $Z \in \gamma_{\mathcal{C}}$ related in the following way:

$$
\begin{aligned}
& \Phi_{t_{u}}\left(X_{u}\right)=Z \\
& \Phi_{t_{s}}\left(X_{s}\right)=Z .
\end{aligned}
$$

The algorithm already gives $X_{u}$ and $X_{s}$ in Lindstedt-Poincaré coordinates

$$
\begin{aligned}
X_{u}=\left(\alpha_{1}, \alpha_{2}, \mathcal{C}, \phi\right) & =\left(\epsilon, 0, \mathcal{C}, \phi_{u}\right), \\
X_{s}=\left(\alpha_{1}, \alpha_{2}, \mathcal{C}, \phi\right) & =\left(0, \epsilon, \mathcal{C}, \phi_{s}\right) .
\end{aligned}
$$

From $(13), X_{u}$ belongs to the unstable manifold of the point $\tilde{X}_{-}=\left(0,0, \mathcal{C}, \phi_{u}\right)$. Similarly, $X_{s}$ belongs to the stable manifold of the point $\tilde{X}_{+}=\left(0,0, \mathcal{C}, \phi_{s}\right)$. By assertion (11),

$$
\begin{aligned}
& Z=\Phi_{t_{u}}\left(X_{u}\right) \in W_{\Phi_{t_{u}}\left(\tilde{X}_{-}\right)}^{u} \\
& Z=\Phi_{t_{s}}\left(X_{s}\right) \in W_{\Phi_{t_{s}}\left(\tilde{X}_{+}\right)}
\end{aligned}
$$


Thus the points we seek are

$$
\begin{aligned}
& X_{-}=\Phi_{t_{u}}\left(\tilde{X}_{-}\right)=\lambda_{t_{u}}\left(\tilde{X}_{-}\right) \\
& X_{+}=\Phi_{t_{s}}\left(\tilde{X}_{+}\right)=\lambda_{t_{s}}\left(\tilde{X}_{+}\right) .
\end{aligned}
$$

In coordinates, they are given by

$$
\begin{aligned}
& X_{-}=\left(0,0, \mathcal{C}, \phi_{-}\right)=\left(0,0, \mathcal{C}, \phi_{u}+t_{u} \omega\right), \\
& X_{+}=\left(0,0, \mathcal{C}, \phi_{+}\right)=\left(0,0, \mathcal{C}, \phi_{s}+t_{s} \omega\right)
\end{aligned}
$$

Therefore,

$$
\begin{aligned}
& \phi_{-}=\phi_{u}+t_{u} \omega, \\
& \phi_{+}=\phi_{s}+t_{s} \omega,
\end{aligned}
$$

so that $\Delta=\phi_{+}-\phi_{-}=\phi_{s}-\phi_{u}+\left(t_{s}-t_{u}\right) \omega(\bmod 2 \pi)$.

\subsection{Some numerical results}

Using the method described in the previous section, we have computed the scattering maps associated to several homoclinic families in the planar restricted three body problem. The results presented here are for Lyapunov orbits around $L_{1}$ in the Earth-Moon model.

Recall from section 3.3 that the scattering map $S^{j, i}$ associated to a given homoclinic family $H^{j, i}$ is characterized by a scalar function $\Delta^{j, i}(\mathcal{C})$ defined on a domain $\mathcal{C} \in\left(\mathcal{C}_{-}^{j, i}, \mathcal{C}_{+}^{j, i}\right)$. The function $\Delta^{j, i}(\mathcal{C})$ is the rotation angle that results from applying $S^{j, i}$ to any point in $\lambda_{\mathcal{C}}$.

Consider the simplest homoclinic families $H^{1,1}$ and $H^{1,2}$, which loop once around the small primary. Figures 6 and 7 show $\Delta^{1,1}$ respectively $\Delta^{1,2}$ in the same domains where the homoclinic families were computed (compare to figure 4). We also plot both functions together in Figure 8 to show how they are a natural continuation of each other. In this way it is also easier to compare the intervals of Jacobi constants where $\Delta^{j, i}$ are defined. The functions are represented in the universal cover, instead of $[0,2 \pi)$, to emphasize that each one is continuous.

Note that the slope of $\Delta(\mathcal{C})$ goes to infinity at the bifurcation point. Note also that $\Delta^{1,2}(\mathcal{C})$ is a twist map for all $\mathcal{C} \in\left(\mathcal{C}_{-}^{1,2}, \mathcal{C}_{+}^{1,2}\right)$ except for a point where $\Delta^{1,2}$ attains a maximum, giving rise there to a non-twist map [4].

\subsection{Example application to Astrodynamics}

This section describes an example application of the scattering map to the design of spacecraft trajectories in the restricted three body problem. The goal of this example is to illustrate the convenience and usefulness of the scattering map as a tool to study homoclinic and heteroclinic phenomena in dynamical systems. 


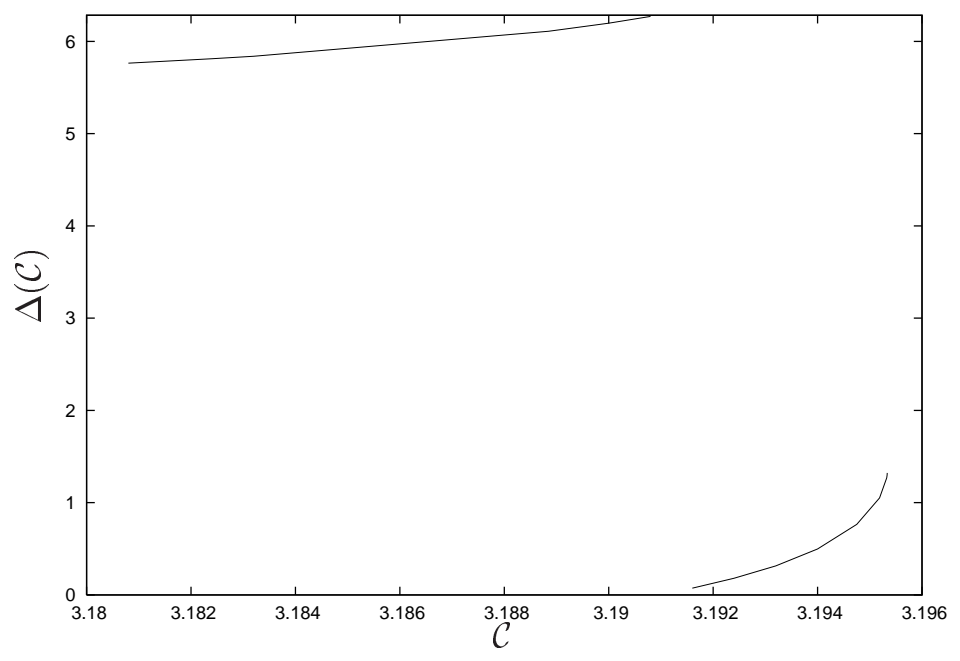

Figure 6: Rotation angle associated to $S^{1,1}$.

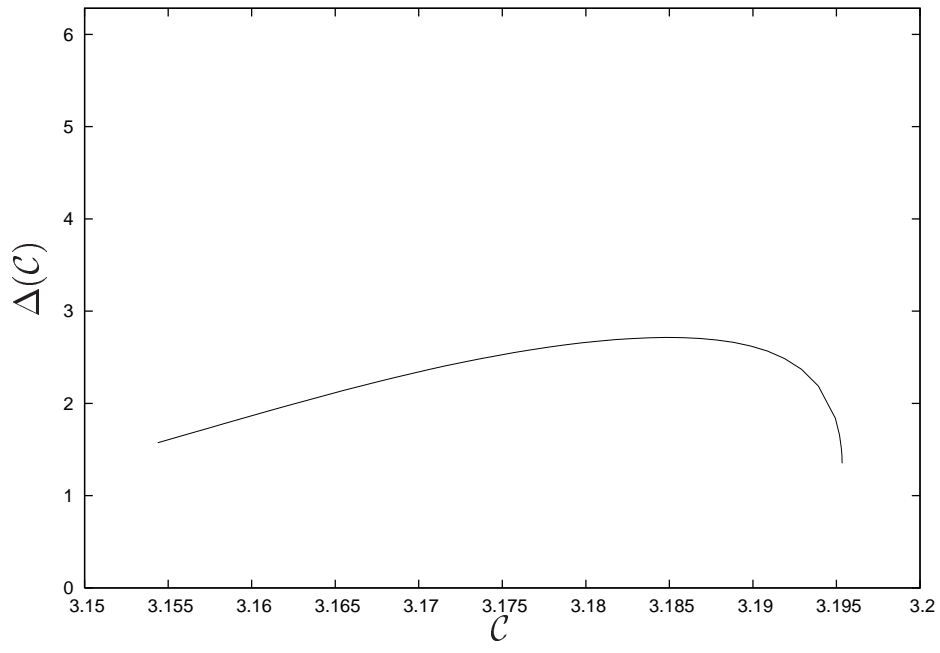

Figure 7: Rotation angle associated to $S^{1,2}$. 


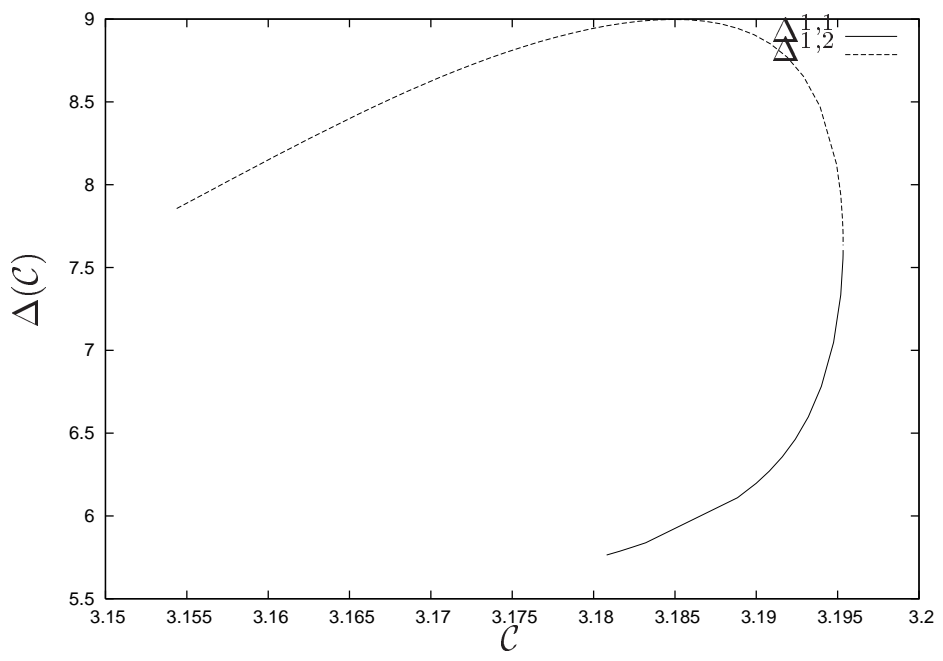

Figure 8: Rotation functions associated to $S^{1,1}$ (solid line) and $S^{1,2}$ (dotted line).

Other applications can probably be found in the field of mission design or in a different field.

As a simplified example using our planar restricted three body problem, let us consider two spacecraft orbiting in a given Lyapunov orbit $\lambda$ about $L_{1}$ in the Earth-Moon system. For any reason (for instance due to a contingency plan or because of launch dispersion) the two spacecraft ended up in a circular like choreography at very different phases in the Lyapunov orbit, while the actual purpose would be to have them close to each other (for instance because of a formation flight requirement). In this situation, performing the rendezvous and overcoming "the gap" between the satellites using classical transfer maneuvers is expensive in terms of delta- $v$ but, using homoclinic connections and with the help of the scattering map, it can be performed at almost zero cost.

The main drawback of this method is that the transfer times are longer than when using classical manoeuvres.

In our example, let us fix some Lyapunov orbit for definiteness, say $\lambda=$ $\lambda_{\mathcal{C}}$ with $\mathcal{C}=3.19$. There are several scattering maps $S^{H}$ defined on $\lambda \subset \Lambda$, depending on the choice of homoclinic family $H$. Recall from section 3.3 that $\lambda$ is parametrized by an angle $\phi$, and the scattering map restricted to $\lambda$ is just a rigid rotation

$$
\phi \mapsto \phi+\Delta .
$$

Having computed several scattering maps numerically in section 3.4, we know the values of $\Delta$ for several homoclinic manifolds. Table 1 shows these values (in radians).

Each application of the scattering map $S^{H}$ corresponds to performing a 
Table 1: Rotation angle for several homoclinic families for $\mathcal{C}=3.19$.

$\begin{array}{ccccccccccc}\Delta^{1,1} & \Delta^{1,2} & \Delta^{2,3} & \Delta^{2,4} & \Delta^{3,1} & \Delta^{3,2} & \Delta^{3,3} & \Delta^{3,4} & \Delta^{4,1} & \Delta^{4,2} & \Delta^{4,4} \\ 6.197 & 2.615 & 2.529 & 5.767 & 2.437 & 5.611 & 5.662 & 2.702 & 5.594 & 2.578 & 5.876\end{array}$

homoclinic excursion through a trajectory $\gamma \subset H$. In the example application, a homoclinic excursion consists in injecting a satellite into the unstable manifold of the Lyapunov orbit using station keeping like maintenance maneuvers, and then following the selected homoclinic trajectory, which naturally comes back to the Lyapunov orbit following its stable manifold. The result is that the satellite is shifted by an angle $\Delta$ with respect to its initial phase.

Therefore, the example problem can be formulated as follows: Let $\mathcal{I}$ be an index set that will be used to index the finite set of scattering maps (or rotation angles) shown in table 1. Equivalently, $\mathcal{I}$ also indexes the homoclinic manifolds considered. Suppose that $x_{A}=\left(\mathcal{C}, \phi_{A}\right)$ and $x_{B}=\left(\mathcal{C}, \phi_{B}\right) \in \Lambda$ are the initial conditions of the two spaceships on the Lyapunov orbit. Find a sequence $\left(i_{1}, i_{2}, \ldots, i_{n}\right) \in \mathcal{I}^{n}$ such that

$$
\operatorname{dist}\left(S^{i_{n}} \circ \cdots \circ S^{i_{2}} \circ S^{i_{1}}\left(x_{A}\right), x_{B}\right)<d
$$

or equivalently

$$
\operatorname{dist}\left(\phi_{A}+\Delta^{i_{1}}+\Delta^{i_{2}}+\cdots+\Delta^{i_{n}}, \phi_{B}\right)<d,
$$

where $d$ is a prescribed distance.

This would correspond to $n$ homoclinic excursions for spaceship $A$. Obviously, one could apply the scattering map to both $\phi_{A}$ and $\phi_{B}$, i.e. applying manoeuvres to both spaceships.

\section{Acknowledgements}

This research has been supported by the Spanish grant BFM2003-09504, and the Catalan grants CIRIT 2001SGR-70 and 2003XT-00021. E.C. Acknowledges the fellowship AP2002-1409 of the Spanish Government. P.R. thanks R. de la Llave for his insightful comments on the scattering map.

\section{References}

[1] J.V. Breakwell, A.A. Kamel, and M.J. Ratner. Station-keeping for a translunar communication station. Celestial Mech., 10:357-373, 1974.

[2] E. Canalias and J.J. Masdemont. Homoclinic and Heteroclinic Transfer Trajectories between Lyapunov Orbits in the Sun-Earth and Earth-Moon Systems. Discrete Contin. Dyn. Syst., 14:261-279, 2006. 
[3] E. Canalias and J.J. Masdemont. IAC-05-C1.1.07, Lunar Space Station for providing services to solar Libration Point Missions. 56th. International Astronautical Federation Congress, Fukuoka, Japan. 17-21 October, 2005.

[4] A. Delshams and R. de la Llave. KAM theory and a partial justification of Greene's criterion for nontwist maps. SIAM J. Math. Anal., 31(6):1235$1269,2000$.

[5] A. Delshams, R. de la Llave, and T.M. Seara. A geometric mechanism for diffusion in Hamiltonian systems overcoming the large gap problem: heuristics and rigorous verification on a model. Mem. Amer. Math. Soc., 2005 , in press.

[6] R.W. Farquhar. A Halo Orbit Lunar Station. Astronautics and Aeronautics., 10(6):52-63, 1972.

[7] G. Gomez, A. Jorba, J.J. Masdemont, and C. Simo. A Dynamical Systems Approach for the Analysis of the SOHO Mission. Third International Symposium on Spacecraft Flight Dynamics. ESA/ESOC., pages 449-454, October, 1991.

[8] J. J. Masdemont. High-order expansions of invariant manifolds of libration point orbits with applications to mission design. Dyn. Syst., 20(1):59-113, 2005 .

[9] C. Robinson. Dynamical systems. Studies in Advanced Mathematics. CRC Press, Boca Raton, FL, second edition, 1999. Stability, symbolic dynamics, and chaos.

[10] V. Szebehely. Theory of orbits. Academic Press, 1967. 